Copyright by the American Physical Society. Chen, Y. C.; Di Ventra, M., "Shot noise in nanoscale conductors from first principles," Phys. Rev. B 67, 153304 DOI: http://dx.doi.org/10.1103/PhysRevB.67.153304

PHYSICAL REVIEW B 67, 153304 (2003)

\title{
Shot noise in nanoscale conductors from first principles
}

\author{
Yu-Chang Chen and Massimiliano Di Ventra* \\ Department of Physics, Virginia Polytechnic Institute and State University, Blacksburg, Virginia 24061
}

(Received 28 February 2003; published 28 April 2003)

\begin{abstract}
We describe a field-theoretic approach to calculate quantum shot noise in nanoscale conductors from first principles. Our starting point is the second-quantization field operator to calculate shot noise in terms of single quasiparticle wave functions obtained self-consistently within the density-functional theory. The approach is valid in both linear and nonlinear response and is particularly suitable in studying shot noise in atomic-scale conductors. As an example, we study shot noise in Si atomic wires between metal electrodes. We find that shot noise is strongly nonlinear as a function of bias and it is enhanced for one- and two-Si wires due to the large contribution from the metal electrodes. For longer wires it shows an oscillatory behavior for even and odd number of atoms with opposite trend with respect to the conductance, indicating that current fluctuations persist with increasing wire length.
\end{abstract}

DOI: 10.1103/PhysRevB.67.153304

PACS number(s): 75.10.Jm, 75.20.Hr

The subject of steady-state current fluctuations (shot noise) has attracted much attention from both theorists and experimentalists over the past decade. ${ }^{1}$ Shot noise is due to charge quantization, and, as such, is generally unavoidable even at zero temperature. While in signal processing, shot noise might be considered as an undesirable effect that blurs the signal detection, in mesoscopic and nanoscale systems it is a physical mechanism that can provide useful information to probe the electron energy distribution, ${ }^{2}$ the kinetics of electrons, ${ }^{3}$ and electron interactions due to the Coulomb repulsion and the Pauli exclusion principle. ${ }^{4}$

When electrons in a conductor diffuse in a completely uncorrelated way, the shot-noise magnitude reaches the wellknown Poisson limit 2eI, where $e$ is the electron charge and $I$ is the dc current. ${ }^{5}$ In all other cases, shot noise is proportional to the average current times a real number $F$, called Fano factor. ${ }^{1}$ Except for some special cases such as transport in resonant-tunneling diodes, where shot noise is significantly enhanced in the negative differential resistance region, due to enhanced electron interaction in the well, ${ }^{6}$ the Fano factor is generally lower than one. ${ }^{7-14}$ In a two-terminal conductor with $n$ channels, the Fano factor can be expressed in linear response and at zero temperature in terms of the transmission coefficients $T_{n}$ of each channel $n^{15-17}$

$$
F=\sum_{n} T_{n}\left(1-T_{n}\right) / \sum_{n} T_{n}
$$

where the denominator is simply proportional to the average current. In principle, if the transmission probabilities $T_{n}$ 's are known, shot noise can be evaluated using Eq. (1). However, the transmission probabilities, and consequently shot noise, are an inter-related function of the electronic and ionic distributions which are generally nonlinear in the external bias. ${ }^{18-20}$ These distributions need to be calculated selfconsistently at all external voltages, and cannot be simply derived from ground-state calculations. ${ }^{18,20}$ The last point is particularly relevant in nanoscale conductors where the chemistry of single atoms is extremely important in determining the current-voltage characteristics of the whole sample. $^{18}$
In this paper we describe a field-theoretic approach to calculate quantum shot noise in nanoscale conductors, where the electronic and ionic distributions are calculated selfconsistently. In particular, we derive an expression of shot noise in terms of single-particle wave functions. The latter quantities can then be determined using a scattering approach within the density-functional theory of many-electron systems. ${ }^{21}$ As an example, we apply the method to the study of shot noise in $\mathrm{Si}$ atomic wires between metal electrodes. We find that shot noise is strongly nonlinear as a function of bias and it is enhanced for very short wires due to the large contribution from the metal electrodes, while for longer wires it shows an oscillatory behavior for even and odd number of atoms. ${ }^{22}$

Let us start by writing the field operator of propagating electrons for a sample connected to a left $(L)$ and right $(R)$ reservoir in terms of single-particle wave functions $\Psi_{E}^{L(R)}\left(\mathbf{r}, \mathbf{K}_{\|}\right)$with energy $E$ and component of the momentum parallel to the electrode surface $\mathbf{K}_{\|}^{21}$ (see Fig. 1 for a schematic of the system investigated)

$$
\hat{\Psi}=\hat{\Psi}^{L}+\hat{\Psi}^{R},
$$

where

$$
\hat{\Psi}^{L(R)}=\sum_{E} a_{E}^{L(R)}(t) \Psi_{E}^{L(R)}\left(\mathbf{r}, \mathbf{K}_{\|}\right) .
$$

The coefficients $a_{E}^{L(R)}(t)=\exp (-i \omega t) a_{E}^{L(R)}$ are the annihilation operators for electrons incident from the left (right) reservoir, satisfying the usual anticommutation relations: $\left\{a_{E}^{i \dagger}, a_{E^{\prime}}^{j}\right\}=\delta_{i j} \delta\left(E-E^{\prime}\right)$. We assume that the electrons coming from the left (right) electrodes thermalize completely in the reservoirs, i.e., the statistics of electrons coming from the left (right) electrodes is determined by the equilibrium Fermi-Dirac distribution function $f_{E}^{L(R)}$ in the left (right) reservoir, i.e.,

$$
\left\langle a_{E}^{i \dagger} a_{E^{\prime}}^{j}\right\rangle=\delta_{i j} \delta\left(E-E^{\prime}\right) f_{E}^{i},
$$

with $i, j=R, L$. From the above field operator, we can define the current operator 


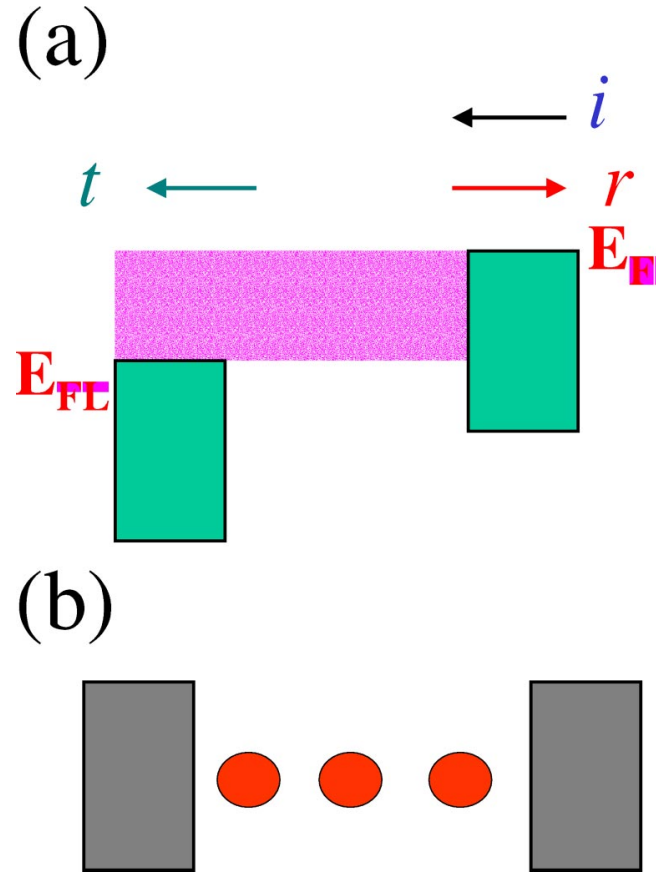

FIG. 1. (a) Energy diagram of the two bare electrodes kept at a certain external potential difference. The left electrode is positively biased: electrons incident from the right electrode are partly transmitted into the left electrode with probability $t$, and reflected back into the right electrode with probability $r$. (b) Schematic of the system investigated; the sample atoms are sandwiched between two bulk electrodes.

$$
\hat{I}(z, t)=-i \int d \mathbf{R} \int d \mathbf{K}_{\|}\left(\hat{\Psi}^{\dagger} \partial_{z} \hat{\Psi}-\partial_{z} \hat{\Psi}^{\dagger} \hat{\Psi}\right),
$$

where $d \mathbf{R}$ defines an element of the electrode surface. For the sake of simplicity, we will assume in the following that the right Fermi level $E_{F R}$ is higher than the left Fermi level $E_{F L}$, so that the average value of the current at zero temperature is simply

$$
\langle\hat{I}\rangle=-i \int_{E_{F L}}^{E_{F R}} d E \int d \mathbf{R} \int d \mathbf{K}_{\|}\left(\widetilde{I}_{E, E}^{R, R}\right),
$$

where

$$
\widetilde{I}_{E, E^{\prime}}^{i j}=\left(\Psi_{E}^{i}\right) * \nabla \Psi_{E^{\prime}}^{j}-\nabla\left(\Psi_{E}^{i}\right) * \Psi_{E^{\prime}}^{j},
$$

with $i, j=R, L$.

We now calculate the shot noise as the Fourier transform of the electric current autocorrelation function in the limit of zero frequency and zero temperature. ${ }^{1}$ The electric current we refer to is the excess current $\Delta \hat{I}$ with respect to the average current Eq. (6). We first consider the spectral density

$$
2 \pi S(\omega)=\int d t e^{i \omega t}\langle\Delta \hat{I}(t) \Delta \hat{I}(0)\rangle
$$

where $\Delta \hat{I}(z, t)=\hat{I}(z, t)-\langle\hat{I}\rangle$ corresponds to the excess (with respect to the average) current operator. We evaluate the quantum statistical expectation value in Eq. (8) by using the Bloch-De Dominicis theorem ${ }^{23}$ (see also Ref. 16) to obtain

$$
\begin{aligned}
S(\omega)= & \sum_{i, j=L, R} \int d E f_{E+\omega}^{i}\left(1-f_{E}^{j}\right) \int d \mathbf{R}_{1} \\
& \times \int d \mathbf{K}_{1} \widetilde{I}_{E+\omega, E}^{i j} \int d \mathbf{R}_{2} \int d \mathbf{K}_{2} \widetilde{I}_{E, E+\omega}^{j i} .
\end{aligned}
$$

In the limit of zero frequency and zero temperature the Fermi distribution function reduces to the step function $f_{E}^{L(R)}$ $=\theta\left(E_{F L(R)}-E\right)$, and the noise power $2 \pi S(\omega=0)$ $=\lim _{T \rightarrow 0} \int d t\langle\Delta \hat{I}(t) \Delta \hat{I}(0)\rangle$ can be written as

$$
S=\int_{E_{F L}}^{E_{F R}} d E\left|\int d \mathbf{R} \int d \mathbf{K} \widetilde{I}_{E, E}^{L R}\right|^{2}
$$

where the range of energy integration is from $E_{F L}$ to $E_{F R}$ and $\tilde{I}_{E, E}^{L R}$ has been defined in Eq. (7).

Equation (10) is the desired expression that relates current fluctuations to the single-particle wave functions. As it was previously noted, it is clear from Eq. (10) that shot noise is not simply proportional to the conductance of the sample ${ }^{1}$ but is determined by an interplay between electron wave functions incident from the left electrode and electron wave functions incident from the right electrode. ${ }^{24}$ It is also interesting to point out that at zero temperature and for a finite bias, there are no electrons incident from the left electrode in the energy region between the left and right Fermi levels (see Fig. 1). The nonzero value of $S$ is thus a direct consequence of quantum-mechanical statistics reflecting the fact that current fluctuations at zero temperature are a purely quantummechanical effect.

Equation (10) can be applied to study the behavior of shot noise in any system once the single-particle wave functions are known. We evaluate these wave functions selfconsistently using the Lippmann-Schwinger equation ${ }^{21}$

$$
\begin{aligned}
\Psi_{E}^{L(R)}\left(\mathbf{r}, \mathbf{K}_{\|}\right)= & \Psi_{0, E, \mathbf{K}_{\|}}^{L(R)}(\mathbf{r}) \\
& +\int d \mathbf{r}_{1} \int d \mathbf{r}_{2} G\left(\mathbf{r}, \mathbf{r}_{1}\right) V\left(\mathbf{r}_{1}, \mathbf{r}_{2}\right) \Psi_{E}^{L(R)}\left(\mathbf{r}_{2}, \mathbf{K}_{\|}\right)
\end{aligned}
$$

where $\Psi_{0, E, \mathbf{K}_{\|}}^{L(R)}(\mathbf{r})=\exp \left(i \mathbf{K}_{\|} \cdot \mathbf{R}\right) u_{E \mathbf{K}_{\|}}^{L(R)}(z)$ are the wave functions corresponding to propagating electrons incident from the left (right) electrode in the absence of the scattering potential $V\left(\mathbf{r}_{1}, \mathbf{r}_{2}\right), G$ is the corresponding Green's function, and $\mathbf{R}$ and $z$ are the coordinates parallel and perpendicular to the electrode surface, respectively. ${ }^{25}$ Deep into the electrodes $(z \rightarrow \pm \infty)$, the wave functions $u_{E \mathbf{K}_{\|}}^{L(R)}(z)$ assume different scattering boundary conditions according to their energy and parallel momentum. ${ }^{21}$ The potential $V\left(\mathbf{r}_{1}, \mathbf{r}_{2}\right)$ describes the difference in potential between the complete system and the bare electrodes. ${ }^{21}$ It is the sum of the nuclear, Hartree, and exchange-correlation potentials. ${ }^{21}$ We choose to represent the latter in the local-density approximation to densityfunctional theory. ${ }^{26}$ Once the single-particle wave functions are calculated self-consistently using Eq. (11), the electric 


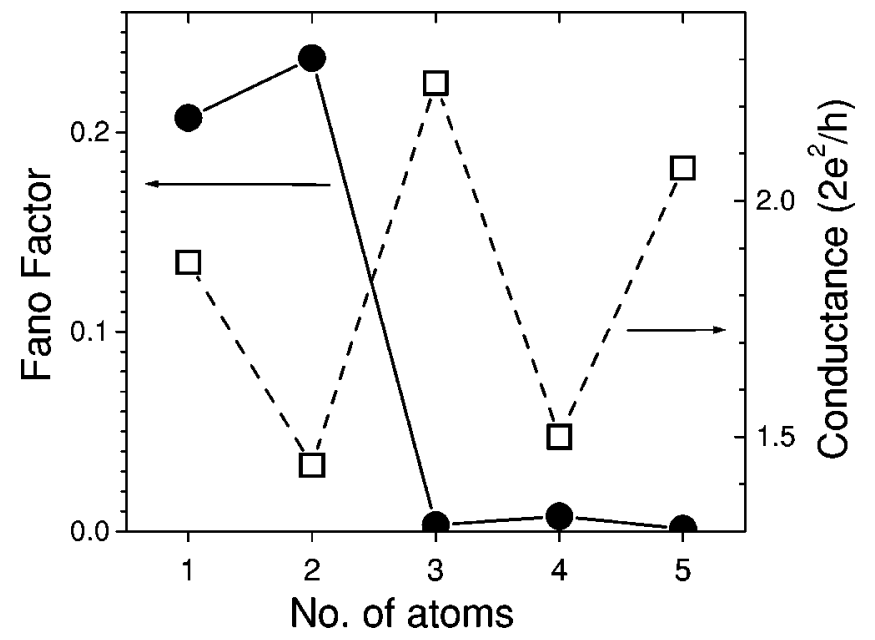

FIG. 2. Dependence of the Fano factor (left axis) and conductance (right axis) on the number of $\mathrm{Si}$ atoms in an atomic wire between two planar electrodes in a linear-response regime (bias $=0.01 \mathrm{~V})$.

current and shot noise can be calculated using Eqs. (6) and (10). Current-induced forces and, consequently, the ionic distribution in steady state can also be determined with the above wave functions. $^{27}$

The current operator of interest to us is the one corresponding to the extra current due to the presence of the sample atoms between the two bare electrodes (see Fig. 1): $\delta \hat{I}(z, t)=\hat{I}(z, t)-\hat{I}_{0}(z, t)$, where the first term on the righthand side is defined in Eq. (5), and the second one is the equivalent term due to the bare electrodes only. In the following, when we discuss about the average current, Eq. (6), and related shot noise, Eq. (10), we refer to the extra current operator $\delta \hat{I}(z, t)$ and corresponding fluctuations. ${ }^{21,27}$ Fluctuations of the extra current are nonlinear in terms of $\hat{I}$ and $\hat{I}_{0}$ implying an enhancement of shot noise due to the contribution from the bare electrodes when $\hat{I}$ and $\hat{I}_{0}$ have comparable magnitude.

As an example of application, we calculate the shot noise for Si atomic wires between two bulk electrodes (see Fig. 1) as a function of the wire length and as a function of bias. The bulk electrodes are modeled with ideal metals (jellium model). ${ }^{21}$ The interior electron density of the electrodes is taken equal to the value for metallic gold $\left(r_{s} \approx 3\right)$. The single-particle wave functions are expanded in plane waves and convergence of both the average current and shot noise has been checked with increasing number of plane waves. ${ }^{21}$ The spacing between the silicon atoms is fixed at 4.2 a.u., and the distance between the electrode edge and the first silicon atom is 2.1 a.u., the relaxed atomic positions at zero bias. $^{27}$ We keep the ionic configuration of the system unchanged at all different biases. In Fig. 2, we plot the Fano factor (defined as $F=S / 2 e I$ ) as a function of the number of $\mathrm{Si}$ atoms for an external bias of $0.01 \mathrm{~V}$ (linear-response regime). In the same plot (right axis), we also show the conductance in units of the quantum of conductance $G_{0}$ $=2 e^{2} / h$. The conductance of the wires oscillates as a function of the number of atoms. The origin of even and odd

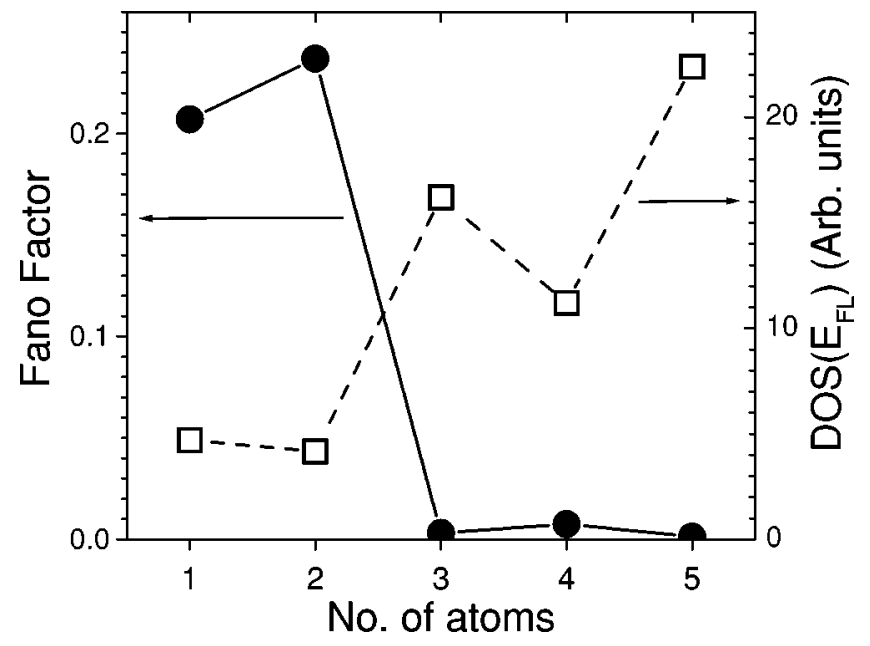

FIG. 3. Dependence of the Fano factor (left axis) and density of states at the left Fermi level (right axis) on the number of Si atoms in an atomic wire between two planar electrodes in a linearresponse regime $($ bias $=0.01 \mathrm{~V})$.

parity oscillations is due to the fact that even-atom-number wires have fully occupied $\pi$ states, while the odd-atomnumber wires have a half-filled $\pi$ state at the Fermi level. This behavior has also been predicted for C-atom wires ${ }^{28}$ that have similar electronic structure. It can be similarly explained in terms of lower (larger) density of states at the Fermi level of the total system (electrodes plus atoms) for even (odd) number of atoms. This is shown in Fig. 3, where the density of states at the left Fermi level (the right Fermi level is at $0.01 \mathrm{eV}$ above the left Fermi level) is plotted as a function of the number of atoms. The Fano factor follows a similar oscillatory pattern. It is lower (larger) for odd (even) number of atoms, exactly opposite to the conductance oscillations behavior. This result can be rationalized by noting that lower conductance implies lower transmission probability and thus larger Fano factor [see Eq. (1)]. These results also indicate that current fluctuations persist with increasing

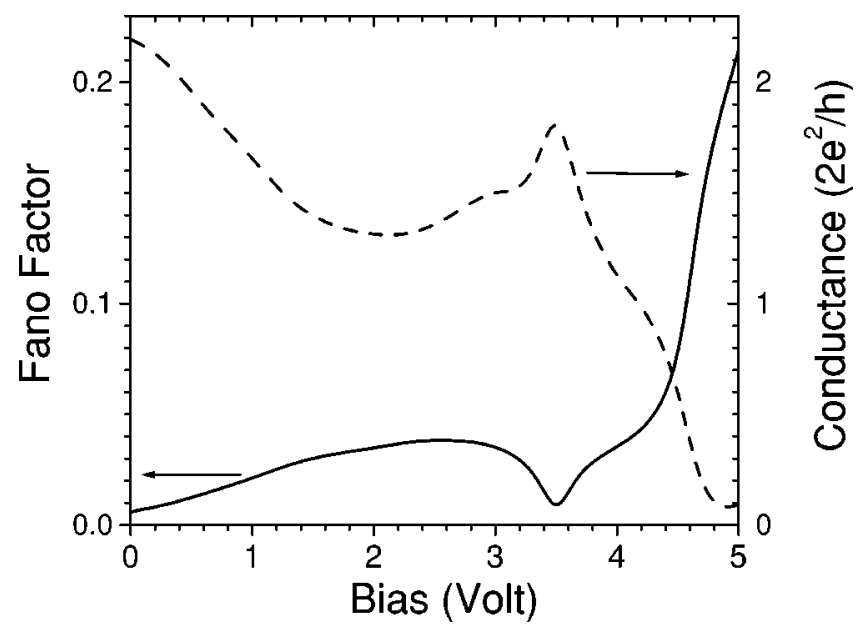

FIG. 4. Differential Fano factor (left axis) and differential conductance (right axis) as a function of applied bias in nonlinear regime for an atomic wire composed of three $\mathrm{Si}$ atoms placed between two electrodes as described in Fig. 1. 
wire length. ${ }^{29}$ For the one- and two-Si atom cases, the Fano factor is enhanced due to the fluctuations introduced by the proximity of the two electrodes. In these two cases, the distance between the electrodes is small so that the current across the electrodes without the $\mathrm{Si}$ atoms inbetween is of the same order of magnitude as the current of the total system (electrodes plus atoms). On the other hand, the shot noise of the bare electrodes without the atoms inbetween is larger than the shot noise of the total system. As a consequence, the Fano factor has a large contribution from the two bare electrodes, and the nonadditive behavior of the total shot noise is evident in this case.

We now discuss the dependence of shot noise on the bias in the nonlinear regime. We choose a three-Si wire as an example. The differential Fano factor [defined as $F$ $=\partial S / \partial(2 e I)]$ as a function of bias is shown in Fig. 4. The differential conductance $(G=\partial I / \partial V)$ is plotted in the same figure (right axis). From Fig. 4, it is evident that the differential conductance reaches a local minimum at about $2 \mathrm{~V}$ and a local maximum at about $3.5 \mathrm{~V}$. The local minimum (maxi- mum) in the conductance is associated to a lower (larger) density of states between the right and left Fermi levels (not shown). The differential Fano factor follows an opposite trend that can be explained again as due to a decreased (increased) transmission probabilities for an electron scattering in the corresponding energy window. The Fano factor is also strongly enhanced in the regime where the differential conductance is close to zero. However, no complete suppression of noise is evident from Fig. 4 at the bias corresponding to the conductance peak. This is due to the contribution of several channels with different $\mathbf{K}_{\|}$that have a transmission probability lower than one at that bias.

We would like to thank Zhongqin Yang for helpful discussions. We acknowledge support from the NSF Grant Nos. DMR-01-02277 and DMR-01-33075, and Carilion Biomedical Institute. Acknowledgement is also made to the Donors of The Petroleum Research Fund, administered by the American Chemical Society, for partial support of this research.
*Email address: diventra@vt.edu

${ }^{1}$ For a recent review, see, e.g., Ya.M. Blanter and M. Büttiker, in Shot Noise in Mesoscopic Conductors, Phys. Rep. 336, 1 (2000).

${ }^{2}$ O.M. Bullashenko and J.M. Rubí, Phys. Rev. E 12, 867 (2002).

${ }^{3}$ R. Landauer, Nature (London) 392, 659 (1998).

${ }^{4}$ R.C. Liu, B. Odom, Y. Yamamoto, and S. Tarucha, Nature (London) 391, 263 (1998).

${ }^{5}$ W. Schottky, Ann. Phys. (Leipzig) 57, 541 (1918).

${ }^{6}$ G. Iannaccone, G. Lombardi, M. Macucci, and B. Pellegrini, Phys. Rev. Lett. 80, 1054 (1998).

${ }^{7}$ L.Y. Chen and C.S. Ting, Phys. Rev. B 43, 4534 (1991); M. Büttiker, Physica B 175, 199 (1991).

${ }^{8}$ A.H. Steinbach, J.M. Martinis, and M.H. Devoret, Phys. Rev. Lett. 76, 3806 (1996).

${ }^{9}$ T. González, C. González, J. Mateos, D. Pardo, L. Reggiani, O.M. Bulashenko, and J.M. Rubí, Phys. Rev. Lett. 80, 2901 (1998).

${ }^{10}$ K.E. Nagaev, Phys. Lett. A 169, 103 (1992).

${ }^{11}$ C.W.J. Beenakker and M. Büttiker, Phys. Rev. B 46, 1889 (1992).

${ }^{12}$ K.E. Nagaev, Phys. Rev. B 52, 4740 (1995).

${ }^{13}$ E.V. Sukhorukov and D. Loss, Phys. Rev. B 59, 13054 (1999); Phys. Rev. Lett. 80, 4959 (1998).

${ }^{14}$ E.G. Mishchenko, Phys. Rev. Lett. 85, 4144 (1998).

${ }^{15}$ G.B. Levovik, JETP Lett. 49, 683 (1989).

${ }^{16}$ M. Büttiker, Phys. Rev. Lett. 65, 2901 (1990).

${ }^{17}$ Th. Martin and R. Landauer, Phys. Rev. B 45, 1742 (1992).

${ }^{18}$ M. Di Ventra, S.T. Pantelides, and N.D. Lang, Phys. Rev. Lett. 84, 979 (2000); Appl. Phys. Lett. 76, 3448 (2000); M. Di Ventra, S.-G. Kim, S.T. Pantelides, and N.D. Lang, Phys. Rev. Lett. 86, 288 (2001); M. Di Ventra, S.T. Pantelides, and N.D. Lang, ibid. 88, 046801 (2002).

${ }^{19}$ Y. Wei, B. Wang, J. Wang, and H. Guo, Phys. Rev. B 60, 16900 (1999).
${ }^{20}$ O.M. Bulashenko and J.M. Rubí, Phys. Rev. B 66, 045310 (2002).

${ }^{21}$ N.D. Lang, Phys. Rev. B 52, 5335 (1995); M. Di Ventra and N.D. Lang, ibid. 65, 045402 (2002); Z. Yang, A. Tackett and M. Di Ventra, ibid. 66, 041405 (2002).

${ }^{22}$ T.-S. Kim and S. Hershfield, Phys. Rev. B 65, 214526 (2002).

${ }^{23}$ R. Kubo, M. Toda, and N. Hashitsume, Statistical Physics II: Nonequilibrium Statistical Mechanics (Springer-Verlag, New York, 1992).

${ }^{24}$ Note that the expression of shot noise Eq. (10) can be recast in terms of transmission coefficients for each conducting channel [see numerator in Eq. (1)]. Y.-C. Chen and M. Di Ventra (unpublished).

${ }^{25}$ Note that the wave functions $\Psi_{E}^{L(R)}\left(\mathbf{r}, \mathbf{K}_{\|}\right)$have the same labeling as the wave functions $\Psi_{0, E, \mathbf{K}_{\|}}^{L(R)}(\mathbf{r})$ even though $\mathbf{K}_{\|}$is no longer a good quantum number. We keep this notation for numerical convenience.

${ }^{26}$ P. Hohenberg and W. Kohn, Phys. Rev. B 136, 864 (1964); W. Kohn and L.J. Sham, Phys. Rev. 140, A1133 (1965); The exchange-correlation functional used in this work is the one of D.M. Ceperley and B.J. Alder, Phys. Rev. Lett. 45, 566 (1980); This is parametrized by J.P. Perdew and A. Zunger, Phys. Rev. B 23, 5048 (1981).

${ }^{27}$ M. Di Ventra and S.T. Pantelides, Phys. Rev. B 61, 16207 (2000); M. Di Ventra, S.T. Pantelides, and N.D. Lang, Phys. Rev. Lett. 88, 046801 (2002); ibid. 89, 139902 (2002).

${ }^{28}$ N.D. Lang and Ph. Avouris, Phys. Rev. Lett. 81, 3515 (1998).

${ }^{29}$ Note that this result disagrees with the one obtained by Scherbakov et al., Phys. Rev. B 57, 6654 (1998) on quantum wires modeled with hard and soft confining potentials. The main difference being the atomic description of the system in our case, and consequent account of the self-consistent electronic distribution in the wire. 